\title{
Frequency of heterozygous TET2 deletions in myeloproliferative neoplasms
}

\author{
This article was published in the following Dove Press journal: \\ Cancer Management and Research \\ 16 September 2010 \\ Number of times this article has been viewed
}

\author{
Joseph Tripodi' \\ Ronald Hoffman' \\ Vesna Najfeld ${ }^{2}$ \\ Rona Weinberg ${ }^{3}$ \\ 'The Myeloproliferative Disorders \\ Program, Tisch Cancer Institute, \\ Department of Medicine and \\ ${ }^{2}$ Department of Medicine and \\ Pathology, Mount Sinai School of \\ Medicine, ${ }^{3}$ The Myeloproliferative \\ Disorders Program, Cellular Therapy \\ Laboratory, The New York Blood \\ Center, New York, NY, USA
}

\begin{abstract}
The Philadelphia chromosome ( $\mathrm{Ph}$ )-negative myeloproliferative neoplasms (MPNs), including polycythemia vera, essential thrombocythemia, and primary myelofibrosis, are a group of clonal hematopoietic stem cell disorders with overlapping clinical and cytogenetic features and a variable tendency to evolve into acute leukemia. These diseases not only share overlapping chromosomal abnormalities but also a number of acquired somatic mutations. Recently, mutations in a putative tumor suppressor gene, ten-eleven translocation 2 (TET2) on chromosome $4 \mathrm{q} 24$ have been identified in $12 \%$ of patients with MPN. Additionally $4 \mathrm{q} 24$ chromosomal rearrangements in MPN, including TET2 deletions, have also been observed using conventional cytogenetics. The goal of this study was to investigate the frequency of genomic TET2 rearrangements in MPN using fluorescence in situ hybridization as a more sensitive method for screening and identifying genomic deletions. Among 146 MPN patients, we identified two patients $(1.4 \%)$ who showed a common $4 \mathrm{q} 24$ deletion, including TET2. Our observations also indicated that the frequency of TET2 deletion is increased in patients with an abnormal karyotype (5\%).
\end{abstract}

Keywords: TET2, myeloproliferative neoplasms, fluorescence in situ hybridization, cytogenetics

\section{Introduction}

The Philadelphia chromosome negative (Ph-) myeloproliferative neoplasms (MPNs) are a group of clonal hematopoietic stem cell disorders with overlapping clinical and cytogenetic features and a variable tendency to evolve into acute leukemia. The Ph- MPNs include polycythemia vera, essential thrombocythemia, and primary myelofibrosis. The current unifying concept of cytogenetic instability of Ph- MPN is a loss or gain of chromosomal regions. The most frequent recurrent chromosomal abnormalities occurring in the Ph- MPNs include trisomy 9/+9p, deletion (20) (q11q13), deletion of $13 q$, and trisomy of chromosomes 8 and 9 . The prognostic significance of the abnormalities in MPNs remains uncertain. However, recent attempts have been made to classify cytogenetic findings in primary myelofibrosis patients as being associated with favorable [isolated deletion (20q) and 13q, trisomy 9 as well as normal karyotype] outcomes and unfavorable outcomes (all other chromosome abnormalities). The number of patients remains too small and the time of follow-up too limited for a definitive validation of the outcomes associated with chromosomal risk profiles. ${ }^{1}$ Nevertheless, it appears that the presence of cytogenetic abnormalities in primary myelofibrosis is associated with shorter survival, but their independent contribution to patient prognosis remains unknown. ${ }^{2}$
Correspondence: Vesna Najfeld

Tumor Cytogenetics, Box 1079, The Myeloproliferative Program, Mount Sinai Medical Center, I Gustave L Levy Place, New York, NY 10029, USA

$\mathrm{Tel}+\mathrm{I} 21224|880|$

Fax + I 2129961096

Email vesna.najfeld@mssm.edu 
Besides cytogenetic abnormalities, these diseases also share acquired somatic mutation of JAK2 in exon 14, resulting in a valine to phenylalanine substitution in codon $617, J A K 2 \mathrm{~V} 617 \mathrm{~F}$, present in over $90 \%$ of patients with polycythemia vera, $60 \%$ of patients with essential thrombocythemia, and $50 \%$ of patients with primary myelofibrosis. ${ }^{3-6}$ Although most MPNs carry mutation on one of two alleles, $2.8 \%$ of MPNs acquire $J A K 2 \mathrm{~V} 617 \mathrm{~F}$ on two $J A K 2$ alleles, ${ }^{7}$ and $3 \%$ of $J A K 2 \mathrm{~V} 617 \mathrm{~F}$-negative patients have mutations in exon 12 of $J A K 2 .{ }^{8}$ Coexistence of these two mutations have also been described. ${ }^{9} M P L$ mutations leading to $M P L W 515 \mathrm{~L}$ occur in $5 \%-10 \%$ of patients with primary myelofibrosis and in $2 \%-5 \%$ of patients with essential thrombocythemia who are negative for JAK2V617F. ${ }^{10,11}$ Other mutations described in MPN patients include mutations of the $C B L$ and $A S X L 1$ genes. ${ }^{12,13}$

Recently, somatic mutations and deletions of the teneleven translocation 2, or TET2, a putative tumor suppressor gene located on chromosome 4, band q24, have been described in $12 \%$ of patients with MPN. ${ }^{14}$ TET2 mutations may predate the $J A K 2 \mathrm{~V} 617 \mathrm{~F}$ mutation, may occur after the acquisition of $J A K 2 \mathrm{~V} 617 \mathrm{~F}$, or may occur simultaneously in two different clones. ${ }^{14,15}$ Following the original description, TET2 nonsense and splice mutations, deletions, and out-of-frame insertions were identified in other myeloid malignancies, including myelodysplastic syndrome (19\%-26\%), de novo acute myeloid leukemia $(12 \%-20 \%)$, therapy-related acute myeloid leukemia (24\%-43\%), chronic myelomonocytic leukemia (20\%-51\%), and systemic mastocytosis (29\%). ${ }^{14,16-24}$ Although the current prognostic value of TET2 mutations in MPNs remains unknown, TET2 mutations were reported in $6 / 14$ patients (43\%) with MPN who had transformed to acute myeloid leukemia. ${ }^{25}$

Deletions of the $4 q 24$ region, including the TET2 gene, have been identified by conventional cytogenetics. In 2005, Viguie et al reported a common deletion of $4 q 24$ in four patients with acute myeloid leukemia/myelodysplastic syndrome. ${ }^{26}$ Additional cytogenetic and high-resolution singlenucleotide polymorphism karyotyping revealed that 20/886 ( $2 \%$ ) patients with myeloid disorders exhibited either partial or complete deletion of TET2. ${ }^{14,18-20}$ In at least four patients with acute myeloid leukemia/myelodysplastic syndrome, the remaining TET2 copy harbored a somatic mutation. ${ }^{19}$ More recently, Hussein compared cytogenetic findings with TET2 status and concluded that unmutated TET2 MPN patients were not cytogenetically different from those MPN patients who harbor TET2 mutations, but there was no information provided regarding deletions of $4 \mathrm{q} 24 /$ TET2. ${ }^{27}$

\section{Materials and methods}

\section{Patients}

A total of 146 patient samples (48 were obtained from the Myeloproliferative Disorders-Research Consortium Tissue Bank) were entered in this study, including myelofibrosis $(n=52)$, polycythemia vera $(n=47)$, essential thrombocythemia $(n=21)$, myeloproliferative neoplasm (unclassified, $\mathrm{MPNu}, \mathrm{n}=20$ ), and $\mathrm{MPNu} /$ myelodysplastic syndrome $(n=6)$. After patient informed consent was obtained, peripheral blood and bone marrow specimens were collected according to the Institutional Review Board guidelines of the Mount Sinai School of Medicine.

\section{Bacterial artificial chromosome clones}

Three bacterial artificial chromosome (BAC) clones, RP11-912N16 (137 kb), RP11-16G16 (175 kb), and RP11-45L9 (162 kb) obtained from BACPAC Resources, Oakland, CA, were grown on chloramphenicol agar plates. The BAC clone RP11-16G16 contains the commonly deleted region on $4 \mathrm{q} 24$, including TET2. To determine the extent of the deletion or possible rearrangements of the 4q24 region, two additional BAC clones, RP11-912N16 and RP11-45L9, containing DNA segments centromeric and telomeric to this commonly deleted region, respectively, were also used. Selected positive colonies were harvested after overnight growth using the Qiagen LargeConstruct kit (Qiagen, Valenica, CA). DNA labeling was achieved using the CGH Nick Translation Kit (Abbott Molecular, Des Plaines, IL) and was fluorescently labeled with spectrum green (RP11-912N16), spectrum aqua (RP11-16G16), and spectrum red (RP11-45L9) according to the Abbott Molecular CGH protocol (Abbott Molecular). Validation fluorescence in situ hybridization (FISH) studies for all three BAC clones were performed on normal controls by evaluating 2000 interphase nuclei. The cutoff percentage for these probes was $3.5 \%$ and was calculated by using the mean \pm three standard deviations. All three BAC probes mapped to the $4 \mathrm{q} 24$ region in 40 examined metaphase cells, demonstrating the $100 \%$ specificity of these probes. In interphase nuclei of normal cells, the expected normal probe signals appear as two tricolor (green, red, aqua) fusions.

\section{Cytogenetic and FISH analysis}

FISH analysis was performed as previously described with pepsin modification treatment $(100 \mathrm{uL} \mathrm{10 \%}$ pepsin and 
$2 \mathrm{~mL} 1 \% \mathrm{HCl}$ ) for five minutes. ${ }^{28}$ Codenaturation of cells and FISH probes were carried out on a Thermobrite (Abbott Molecular) for three minutes at $73^{\circ} \mathrm{C}$ and hybridized at $37^{\circ} \mathrm{C}$ overnight. A total of 200 nuclei were scored in each of the 146 specimens. Metaphase cells were obtained using standard cytogenetic methods. Chromosomal abnormalities were described according to the International System of Human Cytogenetic Nomenclature (2009). ${ }^{29}$

\section{Results}

We investigated 146 patients with $\mathrm{Ph}$ - MPN to determine the frequency of TET2 genomic rearrangements. The summary of FISH studies, using the three TET2 BAC FISH probes as well as the cytogenetic results, are provided in Table 1. Chromosomal analyses were available for 83/146 (57\%) patients; 44 (53\%) patients had a normal karyotype and 39 (47\%) had an abnormal karyotype. The frequency of chromosomal abnormalities among the 39 patients with an abnormal karyotype were: deletion of the long arms of chromosome 20 in 11/39 (29\%), followed by $+9 /+\mathrm{i}(9 \mathrm{p})$ in 9/39 (24\%), trisomy of chromosome 8 in $7 / 39$ (18\%), and trisomy of the long arms of chromosome 1 in 6/39 (16\%). Abnormalities of 4q24 (deletions, translocations, or dupli-

Table I Interphase FISH detection of TET2 deletion

\begin{tabular}{|c|c|c|c|c|c|c|}
\hline & PMF & PV & ET & MPNu & $\begin{array}{l}\text { MPNu/ } \\
\text { MDS }\end{array}$ & Total \\
\hline \multicolumn{7}{|l|}{ Cytogenetics } \\
\hline Normal & 15 & 14 & 6 & 8 & I & 44 \\
\hline Abnormal & 25 & 8 & 2 & 2 & 2 & 39 \\
\hline ND & 12 & 25 & 13 & 10 & 3 & 63 \\
\hline Total & 52 & 47 & 21 & 20 & 6 & 146 \\
\hline \multicolumn{7}{|l|}{$J A K 2$ status } \\
\hline JAK2V6I7F & 4 & 16 & 9 & 0 & 0 & 29 \\
\hline JAK2 wt & 6 & I & 5 & 0 & 0 & 12 \\
\hline ND & 42 & 30 & 7 & 20 & 6 & 105 \\
\hline Total & 52 & 47 & 21 & 20 & 6 & 146 \\
\hline \multicolumn{7}{|l|}{ MPL status } \\
\hline MPLW5I5L & 0 & 1 & I & 0 & 0 & 2 \\
\hline MPL wt & 2 & 4 & 7 & 0 & 0 & 13 \\
\hline ND & 50 & 42 & 13 & 20 & 6 & 131 \\
\hline Total & 52 & 47 & 21 & 20 & 6 & 146 \\
\hline \multicolumn{7}{|l|}{ TET2 status } \\
\hline TET2+ & 2 & 0 & 0 & 0 & 0 & 2 \\
\hline TET2- & 50 & 47 & 21 & 20 & 6 & 144 \\
\hline Total & 52 & 47 & 21 & 20 & 6 & 146 \\
\hline
\end{tabular}

Notes: $(+)$ deletion; (-) I-FISH did not detect deletion of three bacterial artificial chromosomes.

Abbreviations: FISH, fluorescence in situ hybridization; MPNu, myeloproliferative neoplasm (unclassified); ND, not done; wt, wild type; MDS, myelodysplastic syndrome; ET, essential thrombocythemia; PV, polycythemia vera; PMF, primary myelofibrosis. cations) were not detected in any of the 39 patients with an abnormal karyotype by conventional cytogenetics. Interphase FISH analysis using these probes revealed deletion of TET2 in $2 / 83$ patients ( $2 \%$ of total, and $5 \%$ among cytogenetically abnormal).

Patient 34 with primary myelofibrosis was JAK2V617F+ and had a complex karyotype, but 4q24 rearrangement was not identified (see Table 2). Interphase FISH with TET2 revealed a deletion of all three BAC probes in 5\% of interphase nuclei. The follow-up peripheral blood specimen eight months later revealed $30 \%$ of interphase cells with a TET2 deletion, as well as a clone with a cytogenetically identified deletion of the $4 \mathrm{q} 24$ region (see Figure 1). Patient 8 , with primary myelofibrosis, was $J A K 2 \mathrm{~V} 617 \mathrm{~F}+$, had a normal karyotype (see Table 2), and was disomic for the following loci: 7p11.1-q11.1, 8p11.1q11.1, 9p11.1-q11.1, 1q12, 1q21, 1p36, 1q25, 5p15.2, 5q31, 7q31, 9p21 13q14, and 20q12 using interphase FISH. These loci are most frequently rearranged in MPN. In contrast, $90 \%$ of interphase cells revealed a deletion of only the RP1116G16 BAC probe, and not RP11-912N16 and RP11-45L9, indicating a small interstitial TET2 deletion of at least $175 \mathrm{~kb}$ localized between the centromeric and telomeric BAC FISH probes (see Figure 1).

Based on these initial studies, we screened an additional 63 patients for TET2 genomic rearrangements, as shown in Table 1, and none were identified.

Table 1 also shows $J A K 2$ status in 42 patients and $M P L W 515 \mathrm{~L}$ mutational status in 16 patients. Of the three patients who had TET2 deletion, one was MPLW515L mutation-negative, and the $M P L$ mutational status of the remaining two patients was not available.

Among 22,500 cytogenetically examined patients at our institution we identified only three patients with 4q24 chromosomal rearrangements, two with myelodysplastic syndrome and one with primary myelofibrosis (\#34), indicating their rare occurrence. Patient 34 was identified among 512 patients with MPN (polycythemia vera $=361$, myelofibrosis $=151$ ). We therefore used interphase FISH as a more sensitive method to detect 4q24 chromosomal rearrangements and identified an additional patient of the 146 tested patients with TET2 deletion, confirming the published frequency of $1 \%-2 \%$ in MPNs. ${ }^{14,18}$ However, among patients with available conventional cytogenetic results, the frequency was $2 \%$ overall $(2 / 83)$, or $5 \%(2 / 39)$ among the cytogenetically abnormal patients. Two patients had primary myelofibrosis, while none of the 47 patients with polycythemia vera nor 21 patients with essential thrombocythemia had TET2 deletions. 
Table 2 Summary of results from two patients with TET2 deletions

\begin{tabular}{|c|c|c|c|c|c|c|c|c|}
\hline \multirow[t]{2}{*}{ Pt \# } & \multirow[t]{2}{*}{ Dx } & \multicolumn{2}{|c|}{ Mutational status } & \multicolumn{3}{|l|}{ TET2 BACs } & \multirow[t]{2}{*}{$\%$ of nuclei } & \multirow[t]{2}{*}{ Karyotype } \\
\hline & & JAK2V6I7F & MPLW5I5L & RPII-9I2NI6 & RPII-I6GI6 & RPII-45L9 & & \\
\hline 8 & PMF & + & ND & + & - & + & 90 & $46, X Y$ \\
\hline $34^{*}$ & PMF & + & ND & - & - & - & 5 & $\begin{array}{l}45, X Y,-7, \operatorname{del}(\mid 0) \\
(q 2 \mid), \operatorname{inv}(\mid 2) \\
(? p|2 . I ; q| 3.3), \\
\operatorname{der}(\mid 2) t(\mid 2 ; ?), \\
\operatorname{add}(\mid 7)(p \mid I .24)\end{array}$ \\
\hline
\end{tabular}

Notes: On a subsequent follow-up specimen a clone with the karyotype 45,XY,der(4)del(4)(pl4)del(q24),-7,del(8)(p2I)del(I0)(q22),add(I7)(pII.2), del(20(qIIqI3) had developed.

Abbreviations: ND, not done; PMF, primary myelofibrosis; BACs, bacterial artificial chromosomes.

The size of the deletions varied. In one patient (\#8) with a normal karyotype, a deletion of approximately $175 \mathrm{~kb}$ in $90 \%$ of interphase nuclei containing the commonly deleted region of 4q24 (the other two BACs outside the region remained intact) was identified. This observation suggests a submicroscopic (cryptic) deletion beyond the resolution of conventional cytogenetic analysis and confirms the importance of using a higher resolution methodology for identifying TET2 deletion. Moreover, metaphase FISH analysis confirmed a submicroscopic 4q24 deletion that was not detectable by conventional cytogenetics. The other patient (\#34) had a deletion of all three BACs, resulting in a loss of at least $1.6 \mathrm{Mb}$. Interestingly, patient 34 had initially $5 \%$ of interphase cells with TET2 deletion that was not detectable by conventional cytogenetics. When $30 \%$ of his peripheral blood cells showed loss of TET2, a new complex cytogenetic clone emerged showing der(4)del(4)(p14)del(q24), resulting in a complete $4 \mathrm{q} 24$ deletion.

Heterozygous TET2 mutations have been rarely identified in patients with chronic myelogenous leukemia but were detected in those patients who progress to blast crisis or accelerated phase. ${ }^{30}$ Interpretation of these observations suggested that TET2 mutations represent secondary lesions which contribute to the progression of the disease. ${ }^{30} \mathrm{We}$ identified one patient with chronic myelogenous leukemia, not included in this study, who had complex chromosomal aberrations consistent with blast crisis of chronic myelogenous leukemia. FISH analysis revealed a complete deletion of all three BAC probes in 17\% of interphase nuclei.

In summary, TET2 deletions are not a frequent genomic event in MPN. Their deletions are present in about $2 \%$ of MPN patients and, in those with increased karyotype
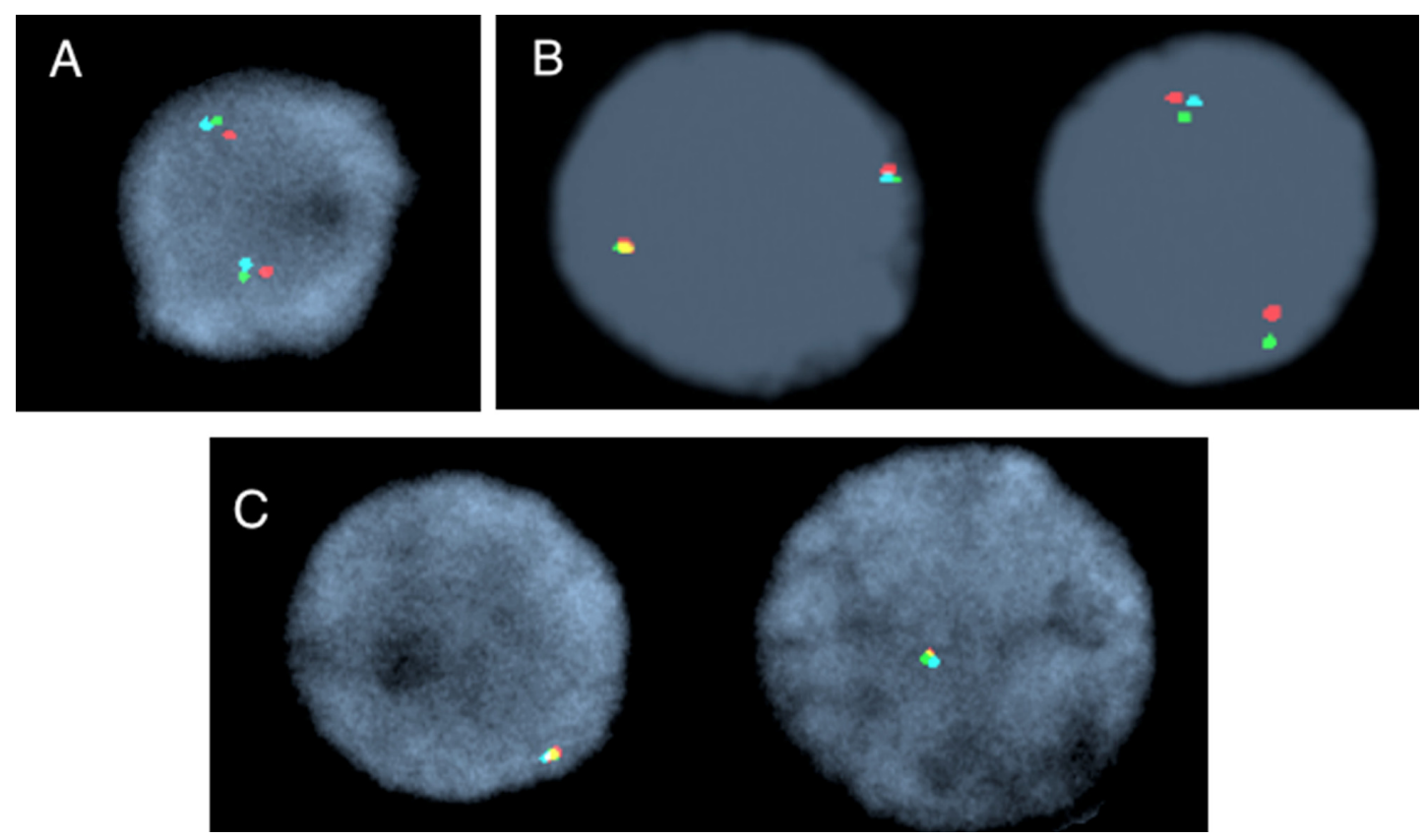

Figure I Interphase nuclei representing TET2 FISH signal patterns. A) Normal nuclei with two triple color fusion signals. B) Deletion of the I75 kb RPI I-I6G I6 BAC probe (aqua). C) Deletion of all three bacterial artificial chromosome probes; note only one triple color fusion signal. 
instability, they may occur in about $5 \%$ of patients. These observations may add supporting evidence that TET2 mutations, as well as deletions, may contribute to leukemic evolution in patients with MPN that transform into leukemia.

\section{Acknowledgment}

This work was supported in part by the P01 CA 108671

MPD-RC grant from the National Institutes of Health.

\section{Disclosure}

The authors report no conflicts of interest in this work.

\section{References}

1. Hussein K, van Dyke DL, Tefferi A. Conventional cytogenetics in myelofibrosis: Literature review and discussion. Eur J Haematol. 2009;82:329-338.

2. Cervantes F, Dupriez B, Pereira A, et al. New prognostic scoring system for primary myelofibrosis based on a study of the International Working Group for Myelofibrosis Research and Treatment. Blood. 2009;113:2895-2901.

3. James C, Ugo V, Le Couédic JP, et al. A unique clonal JAK2 mutation leading to constitutive signalling causes polycythaemia vera. Nature. 2005;434:1144-1148.

4. Kralovics R, Passamonti F, Buser AS, et al. A gain-of-function mutation of JAK2 in myeloproliferative disorders. $N$ Engl $J$ Med. 2005;352:1779-1790.

5. Levine RL, Wadleigh M, Cools J, et al. Activating mutation in the tyrosine kinase JAK2 in polycythemia vera, essential thrombocythemia, and myeloid metaplasia with myelofibrosis. Cancer Cell. 2005; 7:387-397.

6. Baxter EJ, Scott LM, Campbell PJ, et al. Acquired mutation of the tyrosine kinase JAK2 in human myeloproliferative disorders. Lancet. 2005;365:1054-1061.

7. Olcaydu D, Harutyunyan A, Jäger R, et al. A common JAK2 haplotype confers susceptibility to myeloproliferative neoplasms. Nat Genet. 2009;41:450-454.

8. Scott LM, Beer PA, Bench AJ, Erber WN, Green AR. Prevalence of JAK2 V617F and exon 12 mutations in polycythaemia vera. $\mathrm{Br}$ J Haematol. 2007;139:511-512.

9. Li S, Kralovics R, de Libero G, Theocharides A, Gisslinger H, Skoda RC. Clonal heterogeneity in polycythemia vera patients with JAK2 exon12 and JAK2-V617F mutations. Blood. 2008;111:3863-3866.

10. Beer PA, Campbell PJ, Scott LM, et al. MPL mutations in myeloproliferative disorders: Analysis of the PT-1 cohort. Blood. 2008;112: 141-149.

11. Chaligné R, James C, Tonetti C, et al. Evidence for MPL W515L/K mutations in hematopoietic stem cells in primitive myelofibrosis. Blood. 2007;110:3735-3743.
12. Grand FH, Hidalgo-Curtis CE, Ernst T, et al. Frequent CBL mutations associated with $11 \mathrm{q}$ acquired uniparental disomy in myeloproliferative neoplasms. Blood. 2009;113:6182-6192.

13. Carbuccia N, Murati A, Trouplin V, et al. Mutations of ASXL1 gene in myeloproliferative neoplasms. Leukemia. 2009;23:2183-2186.

14. Delhommeau F, Dupont S, Della Valle V, et al. Mutation in TET2 in myeloid cancers. N Engl J Med. 2009;360:2289-2301.

15. Schaub FX, Looser R, Li S, et al. Clonal analysis of TET2 and JAK2 mutations suggests that TET2 can be a late event in the progression of myeloproliferative neoplasms. Blood. 2010;115:2003-2007.

16. Langemeijer SM, Kuiper RP, Berends M, et al. Acquired mutations in TET2 are common in myelodysplastic syndromes. Nat Genet. 2009; $41: 838-842$.

17. Tefferi A, Skoda R, Vardiman JW. Myeloproliferative neoplasms: Contemporary diagnosis using histology and genetics. Nat Rev Clin Oncol. 2009;6:627-637.

18. Jankowska AM, Szpurka H, Tiu RV, et al. Loss of heterozygosity $4 \mathrm{q} 24$ and TET2 mutations associated with myelodysplastic/myeloproliferative neoplasms. Blood. 2009;113:640-710.

19. Kosmider O, Gelsi-Boyer V, Cheok M, et al. TET2 mutation is an independent favorable prognostic factor in myelodysplastic syndromes (MDSs). Blood. 2009;114:3285-3291.

20. Kosmider O, Gelsi-Boyer V, Ciudad M, et al. TET2 gene mutation is a frequent and adverse event in chronic myelomonocytic leukemia. Haematologica. 2009;94:1676-1681.

21. Tefferi A, Lim KH, Levine R. Mutation in TET2 in myeloid cancers. $N$ Engl J Med. 2009;361:1117; author reply 1117, 1118.

22. Tefferi A. Molecular drug targets in myeloproliferative neoplasms: Mutant ABL1, JAK2, MPL, KIT, PDGFRA, PDGFRB and FGFR1. J Cell Mol Med. 2009;13:215-237.

23. Saint-Martin C, Leroy G, Delhommeau F, et al. Analysis of the teneleven translocation 2 (TET2) gene in familial myeloproliferative neoplasms. Blood. 2009;114:1628-1632.

24. Abdel-Wahab O, Mullally A, Hedvat C, et al. Genetic characterization of TET1, TET2, and TET3 alterations in myeloid malignancies. Blood. 2009;114:144-147.

25. Abdel-Wahab O, Manshouri T, Patel J, et al. Genetic analysis of transforming events that convert chronic myeloproliferative neoplasms to leukemias. Cancer Res. 2010;70:447-452.

26. Viguié F, Aboura A, Bouscary D, et al. Common 4q24 deletion in four cases of hematopoietic malignancy: Early stem cell involvement? Leukemia. 2005;19:1411-1415.

27. Hussein K, Abdel-Wahab O, Lasho TL, et al. Cytogenetic correlates of TET2 mutations in 199 patients with myeloproliferative neoplasms. Am J Hematol. 2010;85:81-83.

28. Najfeld V, Montella L, Scalise A, Fruchtman S. Exploring polycythaemia vera with fluorescence in situ hybridization: Additional cryptic $9 \mathrm{p}$ is the most frequent abnormality detected. Br J Haematol. 2002;119:558-566.

29. Shaffer LG, Slovak ML, Campbell LJ editors. ISCN (2009): International System of Human Cytogenetic Nomenclature. Basel: S Karger AG; 2009.

30. Makishima H, Jankowska AM, Cazzolli H, et al. Cbl and TET2 mutations are present in refractory $\mathrm{Ph}+$ disorders including accelerated and blast crisis CML and ALL. Blood. 2009;114:2173.
Cancer Management and Research

\section{Publish your work in this journal}

Cancer Management and Research is an international, peer-reviewed open access journal focusing on cancer research and the optimal use of preventative and integrated treatment interventions to achieve improved outcomes, enhanced survival and quality of life for the cancer patient The journal welcomes original research, clinical \& epidemiological

\section{Dovepress}

studies, reviews \& evaluations, guidelines, expert opinion \& commentary, case reports \& extended reports. The manuscript management system is completely online and includes a very quick and fair peerreview system, which is all easy to use. Visit http://www.dovepress.com/ testimonials.php to read real quotes from published authors. 